PoS $\quad \begin{aligned} & \text { PROCEEDINGS } \\ & \text { OF SCIENCE }\end{aligned}$

\title{
Exploration of the electric spin polarizability of the neutron in lattice QCD
}

\author{
Michael Engelhardt* \\ Department of Physics, New Mexico State University, Las Cruces, NM 88003, USA \\ E-mail: engel@ nmsu.edu
}

\begin{abstract}
A scheme to calculate the electric spin polarizability of the neutron, based on a four-point function approach to the background field method, is presented. The connected contributions to this spin polarizability are evaluated within a mixed action calculation employing domain wall valence quarks on MILC asqtad sea quark ensembles. Results are reported for two pion masses, $759 \mathrm{MeV}$ and $357 \mathrm{MeV}$.
\end{abstract}

XXIX International Symposium on Lattice Field Theory

July 10 - 16, 2011

Squaw Valley, Lake Tahoe, California

${ }^{*}$ Speaker. 


\section{Introduction}

Polarizabilities represent fundamental properties of hadrons, encoding their linear response to externally applied fields. Experimentally, they manifest themselves, e.g., in the non-Born part of the low-energy Compton scattering amplitude. While the leading low-energy response is controlled by the static polarizabilities found in the presence of constant external fields, at subsequent orders of a derivative expansion, the effective hadron Hamiltonian becomes sensitive to temporal and spatial structures in the applied fields. The present study focuses, specifically, on weak, spatially constant external electric fields $\vec{E}$ applied to a neutron. When the fields are sufficiently weak, nonlinear effects, i.e., terms in the effective neutron Hamiltonian of higher order than quadratic in the external field, can be neglected, and, to zeroth order in spatial derivatives as well as second order in temporal derivatives of $\vec{E}$, the neutron mass shift can be expanded as

$$
\Delta m=m(\vec{E})-m(0)=-\frac{1}{2} \alpha_{E} \vec{E}^{2}-\frac{1}{2} \gamma_{E 1} \vec{\sigma} \cdot(\vec{E} \times \dot{\vec{E}})-\frac{1}{2} \alpha_{E v} \dot{\vec{E}}^{2}+\ldots
$$

The static electric polarizability $\alpha_{E}$ has been investigated in a number of lattice studies, cf., e.g., [1-6]. Here, the goal is to perform a first evaluation of the electric spin polarizability $\gamma_{E 1}$ within lattice QCD. An effort in this direction is particularly timely in view of an ongoing experimental program at $\mathrm{HI} \gamma \mathrm{S}$ to measure this polarizability in isolation for the first time, albeit in a proton; previously, only the forward and backward spin polarizabilities $\gamma_{0}, \gamma_{\pi}$ of the proton $[7,8]$, which contain $\gamma_{E 1}$ in combination with other polarizabilities, had been accessed experimentally.

\section{External field and four-point function scheme}

Consider a spatially constant external electromagnetic gauge field $A_{i}$ generating an electric field $E_{i}=\partial_{0} A_{i}$. It enters the lattice link variables $U_{i}$ as an additional phase, $U_{i} \rightarrow \exp \left(i a q A_{i}\right) \cdot U_{i}$, where $a$ denotes the lattice spacing and $q$ the quark electric charge matrix. To evaluate only contributions to the neutron mass shift quadratic in $\vec{E}$, it is sufficient to expand the relevant neutron twopoint function in powers of $\vec{E}$ from the outset. Thus, one can perform a fully dynamical calculation using existing unperturbed gauge ensembles: Expanding $\exp \left(i a q A_{i}\right)=1+i a q A_{i}-\left(a^{2} q^{2} / 2\right) A_{i}^{2}+\ldots$ and inserting into the lattice action decomposes the latter into an unperturbed part and an external field-dependent part, $S=S_{0}+S_{\text {ext }}$, where $S_{\text {ext }}$ is essentially a standard $j \cdot A$ coupling of the external field to the vector current ${ }^{1}$. As a consequence, the neutron two-point function expands as

$$
\left\langle N_{\beta}(y) \bar{N}_{\alpha}(x)\right\rangle=\frac{\int[D U][D \psi][D \bar{\psi}] \exp \left(-S_{0}\right)\left(1-S_{e x t}+S_{e x t}^{2} / 2+\ldots\right) N_{\beta}(y) \bar{N}_{\alpha}(x)}{\int[D U][D \psi][D \bar{\psi}] \exp \left(-S_{0}\right)\left(1-S_{\text {ext }}+S_{\text {ext }}^{2} / 2+\ldots\right)}
$$

Performing the quark integrations yields the diagrammatic representation for the contributions to the neutron two-point function quadratic in $\vec{A}$ depicted in Fig. 1. In the present study, only the connected contributions in Fig. 1 were evaluated. The four-point function scheme at which one has thus arrived is particularly suited to isolate the spin polarizability $\gamma_{E 1}$ from the other contributions appearing in (1.1): As will be specified in more detail below, each vertex in Fig. 1 is associated with an external field insertion $\vec{A}=A_{1} \vec{e}_{1}+A_{2} \vec{e}_{2}$; however, in view of (1.1), only contributions $\sim A_{1} A_{2}$ are of interest, which can be isolated by populating one vertex with only $A_{1}$ and the other vertex

\footnotetext{
${ }^{1}$ Additional contact terms proportional to $A_{i}^{2}$, cf. $[3,4]$, can be excluded in the present context, as seen below.
} 


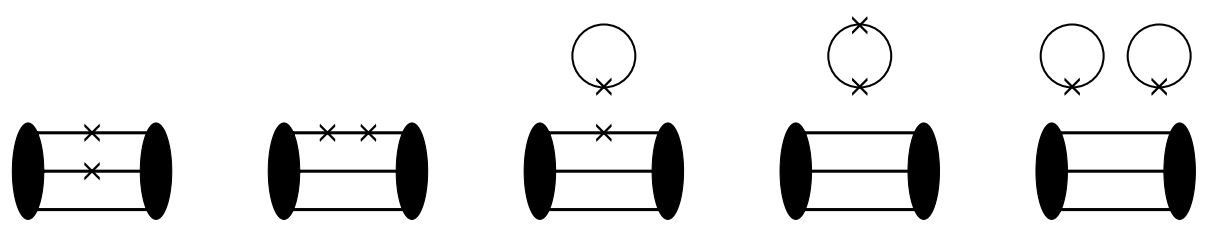

Figure 1: Diagrams contributing to the neutron mass shift; crosses denote $j \cdot A$ insertions.

with only $A_{2}$ in each diagram (and, thus, any contact terms proportional to $A_{i}^{2}$ can also be disregarded, as noted above). This not only discards the signals from the contributions controlled by $\alpha_{E}$ and $\alpha_{E v}$ in (1.1), but also their statistical noise ${ }^{2}$. The very specific control over the different contributions afforded by the present scheme thus aids in reducing the numerical uncertainties.

Choosing the neutron state to be polarized in the 3-direction, a suitable external gauge field is

$$
\vec{A}=\left(a_{1}^{0}+a_{1}^{1} t, a_{2}^{0}+a_{2}^{1} t+a_{2}^{2} t^{2}, 0\right),
$$

which generates a constant $E_{1}$ and an $E_{2}$ varying linearly with time, as called for by (1.1). An ambiguity remains in the choice of the constants $a_{1}^{0}, a_{2}^{0}$ and $a_{2}^{1}$; fixing $E_{1}$ and $\dot{E}_{2}$ only determines $a_{1}^{1}$ and $a_{2}^{2}$. In particular, the finite spatial lattice torus is distinct from an infinite spatial domain in that the constant potentials $a_{i}^{0}$ cannot be wholly eliminated using gauge transformations. Due to the torus boundary conditions, only the residual discrete invariance $A_{i} \longrightarrow A_{i}+2 \pi / q_{\min } L$ remains, where $L$ is the spatial extent of the torus and $q_{\min }$ the smallest unit of electrical charge in the theory. Fractionally different $a_{i}^{0}$ correspond to different physics; namely, they represent flavor-dependent Bloch momenta in view of the minimal substitution $p_{i} \rightarrow\left(p_{i}-q a_{i}^{0}\right)$. Thus, despite the dependences on the constants $a_{1}^{0}, a_{2}^{0}$ and $a_{2}^{1}$ being partially related to one another via space-time symmetries, the neutron mass shift is influenced by additional physics distinct from the spin polarizability effect,

$$
\Delta m=c_{1} \vec{\sigma} \cdot(\vec{A} \times \vec{E})-\frac{1}{2} \gamma_{E 1} \vec{\sigma} \cdot(\vec{E} \times \dot{\vec{E}})
$$

even after having restricted the calculation to contributions $\sim A_{1} A_{2}$. The additional Bloch momentum dependence ${ }^{3}$ must be disentangled from the polarizability effects in order to extract $\gamma_{E 1}$. To this end, several choices of external fields of the type (2.2) will be considered and contrasted below.

\section{Extracting the neutron mass shift in the adiabatic approximation}

In the presence of external fields of the form (2.2), the Hamiltonian of the system under consideration is time-dependent. One facet of this which deserves to be noted is the connection to the Bloch momenta discussed above. One can, e.g., write $A_{1}$ in (2.2) in two equivalent ways: $A_{1}=a_{1}^{0}+a_{1}^{1} t \equiv a_{1}^{1}\left(t-t_{0}\right)$, i.e., a translation of the system by the time $t_{0}$ corresponds to the introduction of a Bloch momentum $a_{1}^{0}=-a_{1}^{1} t_{0}$. This, of course, simply expresses the fact that the electric field $E_{1}$ accelerates or decelerates the Bloch currents. Correspondingly, the Bloch momentum dependence of the neutron mass shift will manifest itself in a characteristic time dependence

\footnotetext{
${ }^{2}$ Note that evaluating the difference between the mass shifts obtained with neutrons polarized in two opposing directions does not achieve this; that eliminates the signal, but not the noise associated with the $\alpha_{E}$ and $\alpha_{E v}$ contributions.

${ }^{3}$ Note that a separate dependence of the form $\vec{A} \times \dot{\vec{E}}$ can be excluded as follows: Working in Euclidean space, each time derivative acquires a factor $i$ compared to Minkowski space. The terms in (2.3) both contain an odd number of time derivatives, and $\Delta m$ will correspondingly be extracted from the imaginary part of the neutron two-point function. By contrast, any putative physical $\vec{A} \times \dot{\vec{E}}$ dependence cannot (and will explicitly be seen not to) arise in the imaginary part.
} 
of the neutron two-point function, as will be seen below.

In general, the time evolution operator for a time-dependent Hamiltonian acquires an intricate time dependence. Here, an adiabatic approximation will be adopted to interpret the measured behavior of the neutron two-point function: Since the external field can be taken to be arbitrarily weak, it seems plausible to consider the case that the strong dynamics equilibrate the system rapidly on the scale of the temporal variation of the external field. In this situation, the behavior of the twopoint function (now projected onto zero momentum states polarized in the 3-direction) is, at times $t$ sufficiently large for excited states to have decayed,

$$
G_{\uparrow}(p=0, t)=\sum_{\vec{y}} \operatorname{Tr}\left(\frac{1+\gamma_{4}}{2}\left(1-i \gamma_{3} \gamma_{5}\right)\langle N(y) \bar{N}(x)\rangle\right)=W \exp \left(-\int d t^{\prime} m\left(t^{\prime}\right)\right)
$$

Inserting expansions of the normalization and the mass ${ }^{4}$ in the external fields (where the superscript specifies the order in the external field),

$$
\begin{aligned}
W & =W_{0}+W^{(1)}[\vec{A}(t)]+W^{(2)}[\vec{A}(t)]+\ldots \\
m & =m_{0}+m^{(2)}[\vec{A}(t)]+\ldots
\end{aligned}
$$

one obtains specifically for the contribution quadratic in the external field

$$
G_{\uparrow}^{(2)}(p=0, t)=W_{0} \exp \left(-m_{0} t\right)\left(\frac{W^{(2)}[\vec{A}(t)]}{W_{0}}-\int d t^{\prime} m^{(2)}\left[\vec{A}\left(t^{\prime}\right)\right]\right)
$$

In view of this, the mass shift $m^{(2)}[\vec{A}(t)]$ can be obtained, after dividing out the unperturbed correlator $G^{(0)}(p=0, t)=W_{0} \exp \left(-m_{0} t\right)$, from the temporal slope of the ratio only at a stationary point of the time evolution, where the time dependences of $W^{(2)}[\vec{A}(t)]$ and $m^{(2)}[\vec{A}(t)]$ are relegated

\begin{tabular}{|c|c|c|c|}
\hline$a m_{l}$ & $a m_{s}$ & $m_{\pi}$ & \# configs \\
\hline \hline 0.01 & 0.05 & $357 \mathrm{MeV}$ & 448 \\
\hline 0.05 & 0.05 & $759 \mathrm{MeV}$ & 425 \\
\hline
\end{tabular}

Table 1: $N_{f}=2+1,20^{3} \times 64$ MILC asqtad ensembles with $a=0.124 \mathrm{fm}$ used in the present investigation. to quadratic order in $t$. Physically, this occurs when the quark Bloch currents cease to flow and turn around into the opposite direction due to the forcing by the external electric field. Only at such a stationary point do the strong dynamics have the opportunity to form a bona fide neutron, the polarizability of which can thus be extracted only in the vicinity of that particular point in time.

\section{Numerical results}

The neutron two-point function was evaluated using the MILC ensembles listed in Table 1. Each configuration was HYP-smeared and chopped into two $20^{3} \times 32$ sublattices with temporal Dirichlet boundary conditions. Domain wall valence quarks were employed. To improve statistics, averages over neutron spin in the positive and negative 3-directions were taken, as well as over the external field (2.2) and its rotation by $\pi / 4$ around the 3-axis. Figs. 2-5 display results for the ratio $R_{2}(t)=G_{\uparrow}^{(2)}(p=0, t) / G^{(0)}(p=0, t)$ for several cases of external field.

Consider first Figs. 2 and 3, which pertain to the case of pion mass $m_{\pi}=759 \mathrm{MeV}$. To exhibit the different effects at play, Fig. 2 (left) shows the result of merely using an external field of the

\footnotetext{
${ }^{4}$ Note that the omission of a linear term $m^{(1)}$ in the ansatz (3.3) implies that the absence of a permanent net electric current or electric dipole moment in an unperturbed neutron has already been put in.
} 

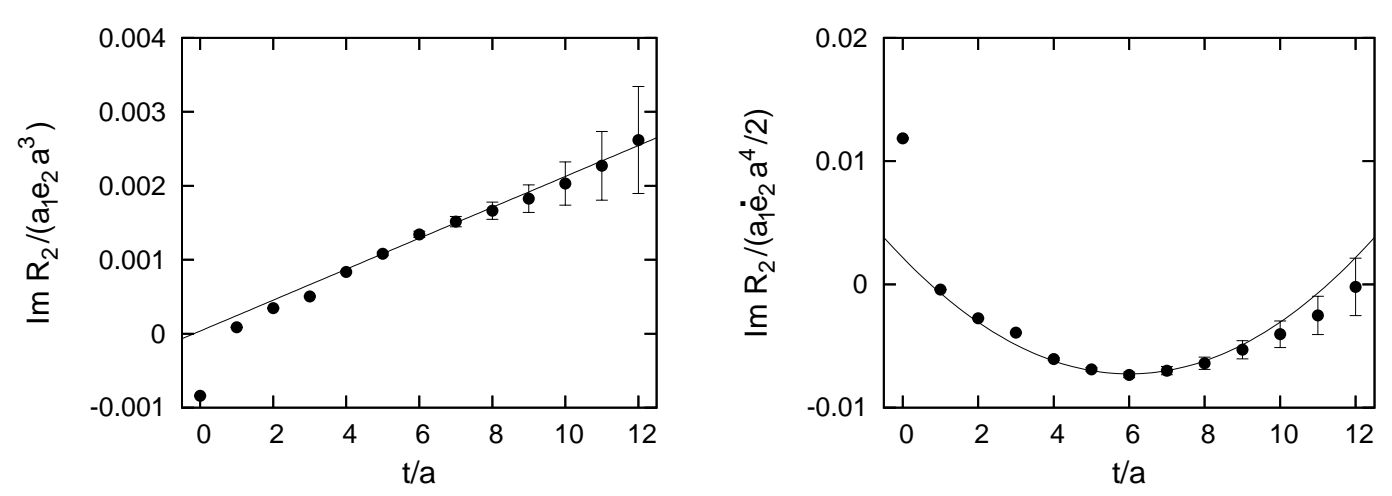

Figure 2: Neutron two-point function ratio $R_{2}$ obtained in the $m_{\pi}=759 \mathrm{MeV}$ ensemble, in the presence of the external fields $\vec{A}=\left(a_{1}, e_{2}\left(t-t_{0}\right), 0\right)$ (left) and $\vec{A}=\left(a_{1}, \dot{e}_{2}\left(t-t_{0}\right)^{2} / 2,0\right)$ (right), where $t_{0}=6 a$. The neutron source is located at $t=0$, electromagnetic fields are in Gaussian units. Linear and quadratic fits, respectively, were performed using the fit range $t / a \in[4,8]$.

form $\vec{A}=\left(a_{1}, e_{2}\left(t-t_{0}\right), 0\right)$, which is expected to yield a time-independent effective dynamics isolating the $\vec{\sigma} \cdot(\vec{A} \times \vec{E})$ dependence in (2.3). Indeed, the correlator ratio $R_{2}(t)$ exhibits linear behavior, corresponding to a constant neutron mass shift determined by the slope of $R_{2}(t)$, cf. (3.4). Continuing with the more complex external field $\vec{A}=\left(a_{1}, \dot{e}_{2}\left(t-t_{0}\right)^{2} / 2,0\right)$, the expected behavior of the correlator ratio $R_{2}(t)$ is parabolic in the vicinity of the stationary point $t=t_{0}$, since now the electric field entering the $\vec{\sigma} \cdot(\vec{A} \times \vec{E})$ dependence of (2.3) is linear in time, crossing zero at $t=t_{0}$. On the other hand, an additional mass shift proportional to $\vec{\sigma} \cdot(\vec{A} \times \dot{\vec{E}})$ would manifest itself as an additional linear term in $R_{2}(t)$, with the effect of shifting the parabola away from $t=t_{0}$. Fig. 2 (right), depicting the corresponding numerical result, corroborates the expected behavior; there is no shift of the parabolic time dependence away from $t=t_{0}$, and therefore no additional dependence on the combination $\vec{\sigma} \cdot(\vec{A} \times \dot{\vec{E}})$, as expected on general grounds, cf. footnote 3 further above. One can moreover verify that the quadratic coefficient of the parabola in Fig. 2 (right) is compatible with the slope in Fig. 2 (left) within numerical error, consistent with the above interpretation.

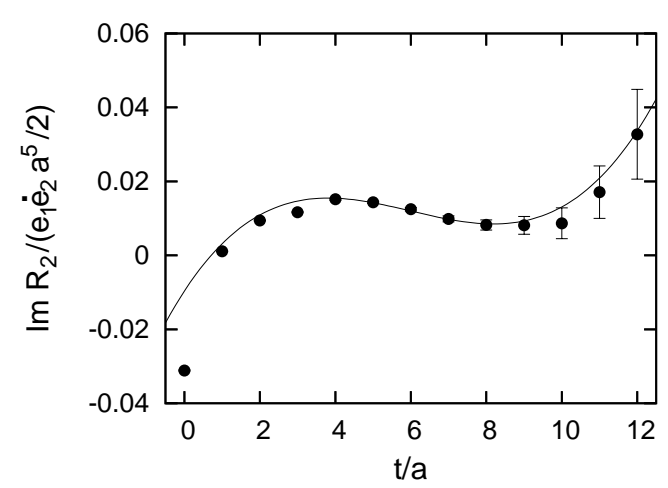

Figure 3: Neutron two-point function ratio $R_{2}$ obtained in the $m_{\pi}=759 \mathrm{MeV}$ ensemble, given the external field $\vec{A}=\left(e_{1}\left(t-t_{0}\right), \dot{e}_{2}\left(t-t_{0}\right)^{2} / 2,0\right)$, where $t_{0}=6 a$. The neutron source is located at $t=0$, and electromagnetic fields are in Gaussian units. Cubic fit was performed using the fit range $t / a \in[4,8]$.
Finally, employing the full external field $\vec{A}=$ $\left(e_{1}\left(t-t_{0}\right), \dot{e}_{2}\left(t-t_{0}\right)^{2} / 2,0\right)$, one would expect to see cubic behavior of $R_{2}(t)$ around the stationary point $t=t_{0}$, since now the time dependences of $A$ and $E$ in $\vec{\sigma} \cdot(\vec{A} \times \vec{E})$ combine to render the latter proportional to $\left(t-t_{0}\right)^{2}$. If this were the only contribution to the neutron mass shift, the slope of $R_{2}(t)$ at the inflection point $t=t_{0}$ would vanish; on the other hand, an additional contribution to the mass shift proportional to $\vec{\sigma} \cdot(\vec{E} \times \dot{\vec{E}})$ would manifest itself as an additional linear term in $R_{2}(t)$, the slope of which could therefore be read off at the inflection point of the overall cubic behavior. This slope, of course, determines the electric spin polarizability $\gamma_{E 1}$ of the neutron 

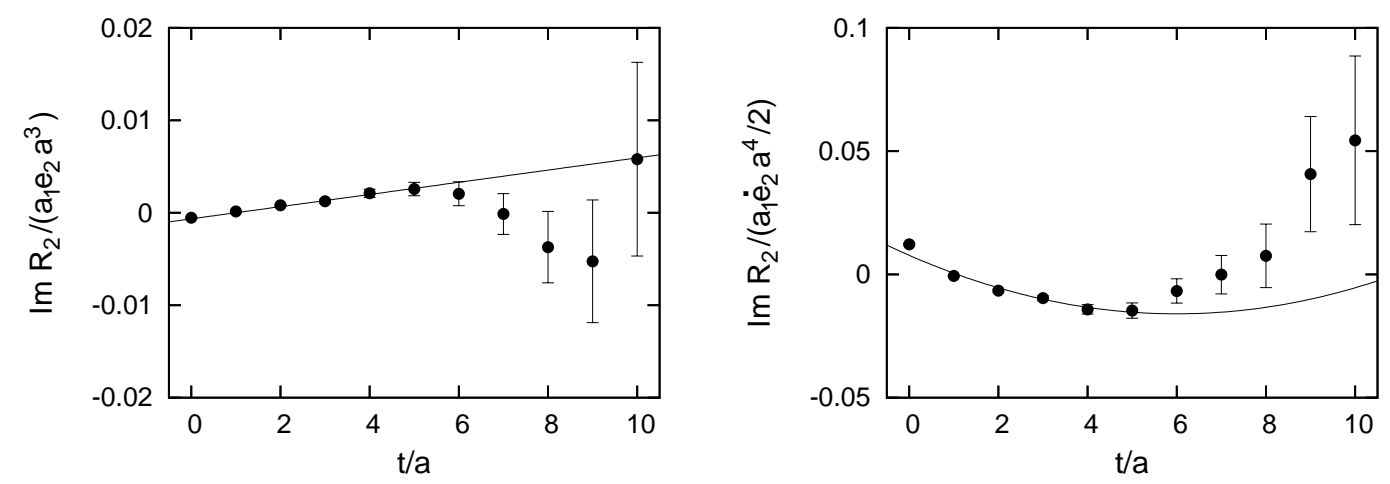

Figure 4: Correlator ratios $R_{2}$ analogous to Fig. 2, except in the $m_{\pi}=357 \mathrm{MeV}$ ensemble, with fits performed using the fit range $t / a \in[3,5]$.

which is the ultimate objective of the analysis. Indeed, the corresponding numerical result for $R_{2}(t)$ shown in Fig. 3 exhibits the expected behavior, including a nonvanishing slope at the stationary point $t=t_{0}$. From this slope, one extracts the value $\gamma_{E 1}=0.0057(8) \cdot 10^{-4} \mathrm{fm}^{4}$, from connected contributions only, at the pion mass $m_{\pi}=759 \mathrm{MeV}$.

Turning to Figs. 4 and 5, which present analogous results for the lighter pion mass $m_{\pi}=$ $357 \mathrm{MeV}$, the principal difference lies in the substantially larger statistical uncertainties, which preclude a clear identification of the functional form of the neutron correlator beyond the time $t=5 a$ in the plots. As a result, fits in a symmetric time range around the stationary point $t=6 a$, as employed in the $m_{\pi}=759 \mathrm{MeV}$ case, are not viable at the present level of statistics. Instead, pending improvement of the numerical accuracy, a fit in the time range $t / a \in[3,5]$ was used to identify the preliminary value $\gamma_{E 1}=0.031(21) \cdot 10^{-4} \mathrm{fm}^{4}$, from connected contributions only, at the pion mass $m_{\pi}=357 \mathrm{MeV}$. Nevertheless, the data depicted in Figs. 4 and 5 display behavior entirely analogous to the one seen in the heavier pion mass case for small times, where the numerical fluctuations are under control.

Finally, Fig. 6 relates the two data points for the spin polarizability $\gamma_{E 1}$ extracted above to partially quenched chiral perturbation theory [9], adjusted to match the present connected diagram calculation. The corresponding expression depends on a number of low energy constants, all but one of which are comparatively well determined from either experiment or previous lattice calcula-

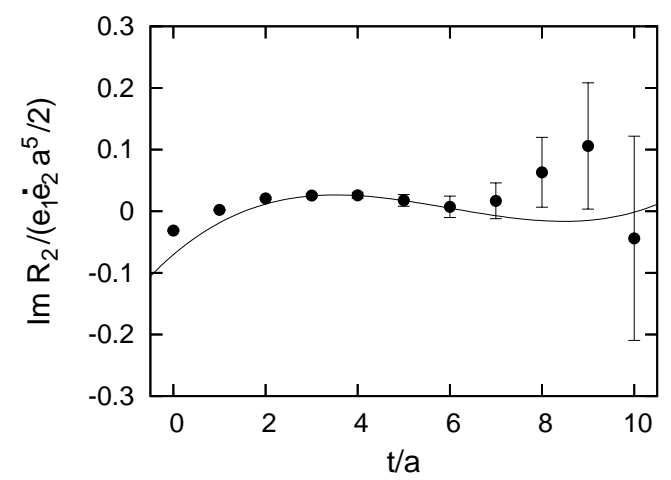

Figure 5: Correlator ratio $R_{2}$ analogous to Fig. 3, except in the $m_{\pi}=357 \mathrm{MeV}$ ensemble, with fit performed using the fit range $t / a \in[3,5]$. tions on the same ensembles. The lone poorly determined parameter, $g_{1}$, has been adjusted such as to generate the two curves in Fig. 6 which pass through the upper and lower ends of the error bar of the $\gamma_{E 1}$ measurement at $m_{\pi}=357 \mathrm{MeV}$. This yields a rather stringent estimate of $g_{1}=-0.15_{-0.01-0.10}^{+0.01+0.04}$, where the first uncertainty quantifies the spread depicted in Fig. 6, and the second one was obtained by varying the other low energy constants within reasonable bounds. Note that errors due to the truncation of the chiral expansion were not estimated. 


\section{Summary}

The present investigation provides first lattice QCD results for the electric spin polarizability $\gamma_{E 1}$ of the neutron, albeit including connected diagrams only. The extraction of this quantity was facilitated by the adopted four-point function approach, which allows for the exact elimination of other contributions to the neutron mass shift in the external electric field considered, namely, ones associated with the static polarizability $\alpha_{E}$ and the dispersion polarizability $\alpha_{E v}$. In addition, the effects of constant external gauge fields, corresponding to quark Bloch momenta on a finite spatial torus, had to be carefully disentangled from the spin polarizability effect itself. A clear signal for

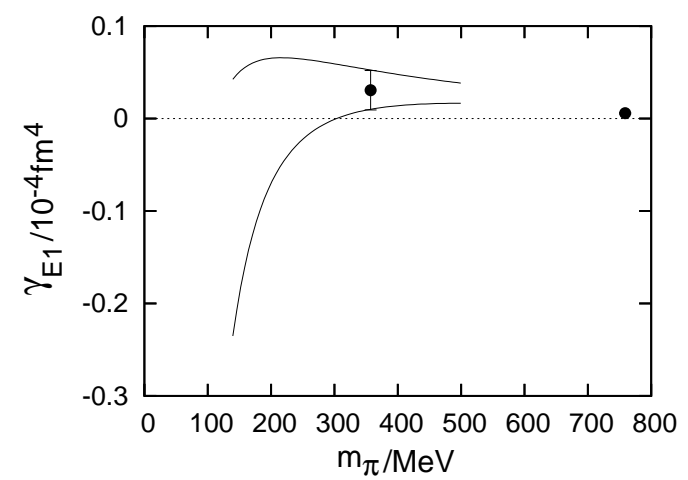

Figure 6: Measured lattice data in relation to partially quenched chiral perturbation theory, cf. text. the connected contribution to $\gamma_{E 1}$ was obtained at the pion mass $m_{\pi}=759 \mathrm{MeV}$; also a preliminary extraction at $m_{\pi}=357 \mathrm{MeV}$ proved possible, although a refinement of the statistical accuracy is desirable. The most striking feature of the connected contribution to $\gamma_{E 1}$ obtained here is its smallness compared to the result expected for full QCD from chiral perturbation theory [9], namely, a negative $\gamma_{E 1}$ larger in modulus by roughly two orders of magnitude. This suggests that the electric spin polarizability $\gamma_{E 1}$ of the neutron is dominated by the disconnected contributions.

\section{Acknowledgments}

Discussions with F. X. Lee, presenting related work at this conference, and W. Detmold are gratefully acknowledged. Computations were performed using Chroma [10] on U.S. DOE/USQCD resources at Fermilab. This work was supported by U.S. DOE grant DE-FG02-96ER40965.

\section{References}

[1] J. Christensen, W. Wilcox, F. X. Lee and L. Zhou, Phys. Rev. D 72 (2005) 034503.

[2] E. Shintani, S. Aoki, N. Ishizuka, K. Kanaya, Y. Kikukawa, Y. Kuramashi, M. Okawa, A. Ukawa and T. Yoshie, Phys. Rev. D 75 (2007) 034507.

[3] M. Engelhardt, Phys. Rev. D 76 (2007) 114502.

[4] M. Engelhardt, PoS LAT2009 (2009) 128.

[5] A. Alexandru and F. X. Lee, PoS LAT2009 (2009) 144.

[6] W. Detmold, B. Tiburzi and A. Walker-Loud, Phys. Rev. D 81 (2010) 054502.

[7] V. Olmos de León et al., Eur. Phys. Jour. A 10 (2001) 207.

[8] J. Ahrens et al. [GDH and A2 Collaborations], Phys. Rev. Lett. 87 (2001) 022003.

[9] W. Detmold, B. Tiburzi and A. Walker-Loud, Phys. Rev. D 73 (2006) 114505.

[10] R. G. Edwards and B. Joó (LHPC and UKQCD Collaborations), Nucl. Phys. Proc. Suppl. 140 (2005) 832. 